\title{
Earth's Multi-scale Topographic Response to Global Mantle Flow
}

\author{
D.R. Davies ${ }^{1}$, A.P. Valentine ${ }^{1}$, S.C. Kramer ${ }^{2}$, N. Rawlinson ${ }^{3}$, M.J. Hoggard ${ }^{4}$, C.M. Eakin ${ }^{1}$, and C.R. Wilson ${ }^{5}$ \\ ${ }^{1}$ Research School of Earth Sciences, The Australian National University, Canberra, Australia. \\ ${ }^{2}$ Department of Earth Science and Engineering, Imperial College London, UK. \\ ${ }^{3}$ Department of Earth Sciences-Bullard Labs, University of Cambridge, Cambridge, UK. \\ ${ }^{4}$ Department of Earth and Planetary Sciences, Harvard University, MA, USA. \\ ${ }^{5}$ Department of Terrestrial Magnetism, Carnegie Institution of Washington, Washington, DC, USA.
}

Earth's surface topography is a direct physical expression of our planet's dynamics. Most is isostatic, controlled by thickness and density variations within the crust and lithosphere, but a significant proportion arises from forces exerted by underlying mantle convection. This dynamic topography directly connects the evolution of surface environments to Earth's deep interior, but predictions from mantle flow simulations are often inconsistent with inferences from the geological record, with little consensus about its spatial pattern, wavelength and amplitude. Here, we demonstrate that previous comparisons between predictive models and observational constraints have been biased by subjective choices. Using measurements of residual topography beneath the oceans, and a hierarchical Bayesian approach to performing spherical harmonic analyses, we generate a robust estimate of Earth's oceanic residual topography power spectrum. This indicates power of $0.5 \pm 0.35 \mathrm{~km}^{2}$ and peak amplitudes of $\sim 0.8 \pm 0.1 \mathrm{~km}$ at long-wavelength $\left(\sim 10^{4} \mathrm{~km}\right)$, decreasing by roughly one order of magnitude at shorter wavelengths $\left(\sim 10^{3}\right.$ $\mathrm{km})$. We show that geodynamical simulations can only be reconciled with observational constraints if they incorporate lithospheric structure and its impact on mantle flow. This demonstrates that both deep (long-) and shallow (shorter-wavelength) processes are crucial, and implies that dynamic topography is intimately connected to the structure and evolution of Earth's lithosphere.

Between Earth's crust and core lies the mantle, a 2,900 km-thick layer of hot rock that constitutes greater than $80 \%$ of Earth's volume. Carrying heat to the surface, the convecting mantle is the 'engine' that drives our dynamic planet: it is directly or indirectly responsible for almost all large-scale tectonic and geological activity [1]. As the mantle flows, it transmits normal stresses to the lithosphere - Earth's rigid outermost shell — that are balanced by gravitational stresses arising through topographic deflections of Earth's surface $[2,3,4,5,6,7,8$, 9]. This so-called dynamic topography is transient, varying both spatially and temporally in response to underlying mantle flow. As a result, it is more challenging to isolate than isostatic topography. The relative importance of dynamic versus isostatic topography varies according to setting: for example, the elevation of the Himalaya is principally isostatic, due to the presence of Earth's thickest continental crust; but the broad excess elevation of the stable South African craton has been 
attributed to dynamic topography, generated by mantle upwelling [10]. Dynamic topography is fundamental to Earth's gravitational field $[5,11]$ and also influences surface processes - including erosion, sediment transport and deposition — as recorded by stratigraphic sequences in sedimentary basins and river profiles $[12,13,14,15,16,17,18]$. It is directly connected to changes in sea level and continental flooding: as continents migrate over areas of positive dynamic topography, large vertical motions lead to the emergence of entire regions; similarly, encountering negative dynamic topography can induce rapid inundation of large areas $[19,20,21,22,23]$. Surface processes may also influence mantle flow: as the topography evolves, the convecting system must respond to maintain a force-balance [24].

Given the importance of dynamic topography, a number of attempts have been made to constrain its spatial pattern, wavelength and amplitude. There are generally two ways to approach this: (i) estimation of so-called residual topography, by removal of the isostatic contribution due to sediments, ice, crust and lithosphere from the observed topography $[25,26$, $27,28,9]$; or (ii) estimation of the surface deflections arising from mantle flow, via computational simulation ('predictive modelling') $[29,30,31,8,32,33,34]$. However, the results obtained using these two approaches are inconsistent. Predictive models generally exhibit peak amplitudes of 1-2 km. They are dominated by broad topographic highs within the Pacific and African domains, separated by a band of topographic lows extending from Antarctica, through the Americas to the Arctic and broadening beneath the Eurasian continent (an example, from [8], is illustrated in Fig. 1a). Residual topography estimates, on the other hand, show smaller-scale structure, with key features including lows at the Australian-Antarctic Discordance (AAD) and Argentine Basin, and highs under the central and western Pacific Ocean, offshore southern Africa, the South China Sea and the North Atlantic (an example, from [9], is displayed in Fig. 1b). The discrepancies between these two approaches are consistently seen across a number of independent studies $[8,35]$ and may arise from a combination of uncertainty on key parameters and approximations made within the analyses, many of which are common across studies. For example, most existing predictive models do not account for the effects of uppermost mantle structure above $225-300 \mathrm{~km}$ depth, owing to the difficulties associated with inferring density from seismic velocity in the vicinity of Earth's highly heterogeneous lithosphere. Furthermore, mantle viscosity and its depth-dependence are a key material property in mantle flow simulations, but estimates vary by at least an order of magnitude. Residual topography estimates also have large uncertainties, principally because the density and thickness of Earth's crust and sedimentary cover, and especially lithospheric thickness variations, are all poorly resolved on a global scale $[8,36,28,35]$. It should also be noted that residual topography estimates cannot often be directly compared to dynamic topography predictions, as the former regularly include unresolved isostatic contributions arising from lithospheric thickness variations, whereas the latter do not.

In an attempt to better constrain residual topography, Hoggard et al. (2016) [9] compiled a database of point-wise estimates within the oceanic realm. At each point, care was taken to remove the isostatic consequences of variable sedimentary loading and crustal thickness from the observed topography, based upon analyses of magnetic anomaly patterns and characteristic acoustic architecture in seismic reflection and refraction profiles. Residual depth anomalies were subsequently calculated by removing the effects of ocean-floor cooling, using an empirical model [37] (although 
isostatic contributions, arising from variations in lithospheric thickness and density unrelated to ocean-floor age, were not removed). To generate a global spherical harmonic decomposition of residual topography, these point-wise constraints were supplemented in the oceans by residual depth measurements from ship-track bathymetry, and on continents by a model that transformed free-air gravity anomalies to residual topography assuming a constant value for admittance. This database, illustrated in Supplementary Fig. 1, was then used to express Earth's residual topography in terms of spherical harmonic functions, using a regularised least-squares inversion algorithm. This allowed the power spectrum of residual topography to be obtained, as illustrated in Fig. 1(e - dotted line). Hoggard et al. (2016) [9] concluded that their dataset could be accurately represented up to and including a maximum spherical harmonic degree of $l=30$, with peak power of $0.1-0.3 \mathrm{~km}^{2}$ at $l=1-3$ (i.e. at wavelengths of $\sim 10,000 \mathrm{~km}$ ) along with significant residual topography, of comparable power, at $l=15$-30 (i.e. at shorter wavelengths of 1,000-2,000 km). In light of the sensitivity kernels that illustrate how effective density anomalies at different depths and spherical harmonic degree are at creating topography $[38,4,5,11,39,9]$, such a spectrum implies a major role for shallow mantle structure and flow. This is inconsistent with most predictive models, which exhibit significant power at $l=2$ and negligible power at shorter wavelengths characteristics that, instead, suggest deep mantle flow as the dominant driver for Earth's surface response [8, 9].

These conclusions have been heavily debated. A number of studies indicate that admittance varies with wavelength and location, with potential for large dynamic topography in the absence of free-air gravity anomalies [38, 11, 32]. This invalidates the continental constraints used by [9], and calls the robustness of their results into question. Nonetheless, if one generates a power spectrum using oceanic residual topography measurements only, or uses a model derived from CRUST 1.0 [40] on continents, the general character of the power spectrum remains consistent [9]. This is also the case using an updated compilation of oceanic (point-wise and ship-track) residual topography measurements, generated herein by building on [41] (see Supplementary Information), where the effects of ocean-floor cooling are removed using a theoretical plate model rather than an empirical model: the spectrum remains reasonably 'flat' (Fig. 1e - solid black line). Taken together, these analyses lend continued support to the conclusions of [9].

In further support of Hoggard et al. (2016) [9], [45] analysed the asymmetric subsidence of mid-ocean ridges, finding only $\sim 500 \mathrm{~m}$ of long-wavelength dynamic topography. Moreover, recently developed models of mantle dynamics, which account for shallow lithospheric structure and small-scale upper mantle convection, display significant power at shorter wavelengths $(l=15-30)[39,46]$, as expected from the aforementioned sensitivity kernels [38, 5, 11, 39, 9]. These models, however, continue to generate long-wavelength residual topography that remains at apparent odds with the observational constraints, displaying significantly more power at $l \leq 2[39,47]$. This is also true of a recent model by Yang \& Gurnis (2016) [32], although they proposed that at least part of the long-wavelength discrepancy arises due to the sparse nature of the observational constraints [41]. Their study also demonstrates that the conclusions of [9] are sensitive to regularisation choices [44] and suggests that the maximum degree to which a spherical harmonic representation can be inferred from the point-wise residual topography measurements is $l=5[32]$. We note that these claims have since been refuted [41] and emphasise that the analyses of $[9,41]$ and $[32,44]$ are not directly comparable: power 
Figures/Fig_1.pdf

Figure 1: Predicted versus inferred topography: (a) simulated present-day dynamic topography from a time-dependent mantle flow simulation [8]; (b) inferred residual topography from observational constraints [9]; (c)/(d) simulated topography from our instantaneous-flow models, neglecting (c) and incorporating (d) shallow mantle and lithospheric structure, respectively; (e) spectral decomposition of published predictive models $[29,30,31,42,8]$ and observation-based residual topography estimates [9, 41]. Note that predictive models cover Earth's surface at high-resolution and have not been regularised, but residual topography estimates have, using an automatic regularisation parameter selection algorithm [43]; (f) unregularised spectral decomposition of our simulations - spectra computed from the predictive models are not directly comparable with the observational constraints, since they omit effects introduced by irregular sampling and processing choices [44]. Comparisons that account for these effects are displayed in Fig. 2.

93 spectra are normalised and scaled differently between studies, and different regularisation approaches are employed; it

94 is therefore not surprising that different conclusions are being drawn. Consequently, we find ourselves at an impasse,

${ }_{95}$ with little agreement on: (i) the spatial pattern, wavelength and amplitude of dynamic topography; and (ii) the relative

96 contributions to dynamic topography from shallow and deep mantle flow. 
In this paper, we employ a new approach to performing spherical harmonic analyses [43], designed to be less dependent on the subjective regularisation choices that have influenced previous studies $[9,41,32,44]$. This allows us to obtain a robust estimate of the power spectrum of Earth's oceanic residual topography field from an updated compilation of the (point-wise and ship-track) dataset of [41]. Through consistent quantitative comparisons between this spectrum and a suite of predictive models of mantle dynamics, we reveal how both deep and shallow mantle flow combine to dictate Earth's oceanic residual topography expression. Finally, using these models as a basis, we isolate the flow-related (truly dynamic) contribution towards Earth's residual topography, by estimating the isostatic effects of mapped lithospheric thickness variations beneath the world's oceans.

\section{The Power Spectrum of Residual Topography}

Our procedure for inferring the power spectrum of topography, closely following that of [9], is described in the Methods. It involves a regularised least-squares inversion of observational constraints to fit a spherical-harmonic expansion to the topographic signal. As with any such approach, the choice of regularisation can have a significant influence on results. To guide such choices, it is helpful to note that the least-squares procedure has a Bayesian interpretation, in which the regularisation operator is identified as the covariance matrix of the prior distribution [48, 43]. Thus, the regularisation operator encodes our assumptions in the absence of any data, and plays a key role in determining the character of any solution. In [9], the regularisation operator was constructed to prefer low-amplitude, smooth solutions. We remark that 'smooth' can be defined in different ways; [9] chose to penalise both the first derivative of the recovered field and the total power contained within it (i.e. the sum of squares of model coefficients). The relative weights assigned to each penalty term in this Tikhonov-style regularisation were governed by two tuneable parameters, which we denote by $\alpha$ (overall power term) and $\beta$ (gradient term), as detailed in the Methods. Although [9] did not adopt an explicitly Bayesian approach, it is instructive to do so, and generate samples from the prior distribution associated with this regularisation. In Supplementary Fig. 2, we show the range of power spectra associated with $10^{6}$ samples, for different $\alpha$ and $\beta$ pairs: regardless of the values adopted, it is clear that this form of regularisation expresses an a priori preference for a relatively 'flat' spectrum. The consequences of this are explored via a series of inversions of synthetic datasets and observational constraints in the Supplementary Information, with key results illustrated in Supplementary Figs. 3 and 4. In short, these tests demonstrate that conclusions are predicated upon the assumptions implicit within the form of regularisation operator, making it difficult to assess whether the power spectrum of [9] reflects signal in the data, or simply the initial biases, as suggested by [44].

To address this issue, we perform the inversion procedure using a different style of regularisation, which we refer to as 'Automatic Relevance Determination' (ARD) [43, 49]. This amounts to introducing one regularisation parameter for each spherical harmonic degree, and then tuning these to match the statistics of the data (see Methods). By doing so, we avoid imposing any constraints upon the expected form of the power spectrum, and allow the data to provide its 


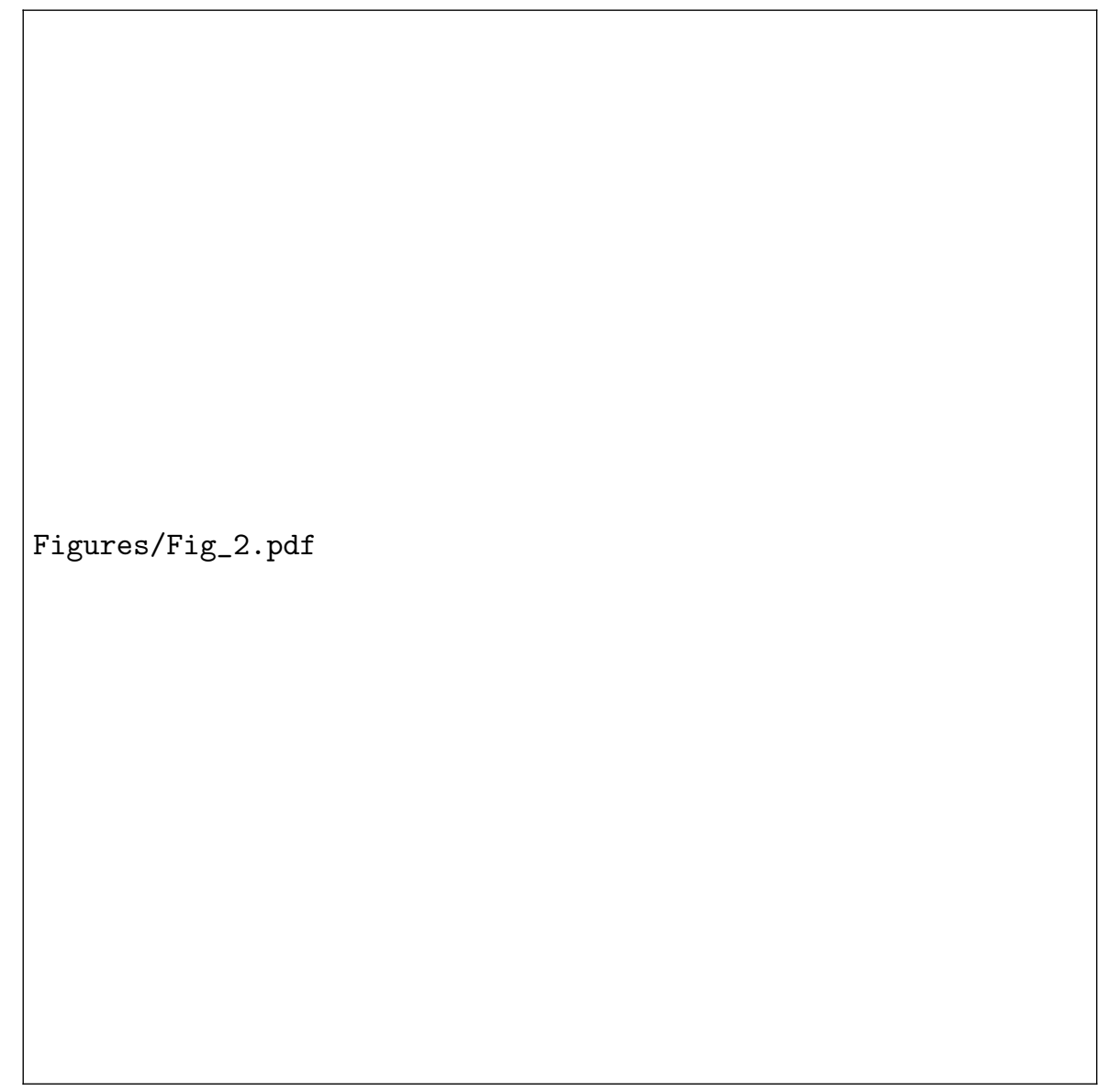

Figure 2: Power spectra obtained from simulated datasets and observational constraints using inversions regularised with Automatic Relevance Determination [43, 49]: solid lines denote results from (simulated) datasets with complete, high-density global coverage; dashed lines represent the mean results obtained using data only at the spot and ship-track locations of observational constraints, whilst shaded regions represent $50 \%$ and $99 \%$ confidence intervals around this mean (see Methods). Red colours denote inversions of a simulated dataset with no shallow structure (Fig. 1c); blue colours depict a simulated dataset with shallow structure present (Fig. 1d); grey colours represent results obtained from the observational constraints. Each dataset has been independently regularised. We see that simulations without shallow structure do not contain evidence for significant power above $l=8$, whereas the observational constraints require these shorter-wavelength features. Although the observational constraints have significantly less power at $l=1$, and slightly less power throughout the remainder of the spectrum, they are broadly compatible with our simulation that contains shallow structure.

own definition of 'smooth'. In Fig. 2, we show results from applying this procedure to the oceanic residual topography dataset, with maps of the resulting spherical harmonic model shown in Supplementary Fig. 5. Our analyses: (i) express a preference for $0.5 \mathrm{~km}^{2}$ of residual topography power at long-wavelength $(l=2)$, likely in the range of $0.25-0.85 \mathrm{~km}^{2}$, with peak amplitudes of $0.8 \pm 0.1 \mathrm{~km}$ - larger than suggested by [9], smaller than predicted by [32, 44], but within error of analyses by [45]; (ii) demonstrate that spectral power decreases by an order of magnitude from $l=2$ to $l=30$; and (iii) support the presence of a low amplitude short-wavelength $(l=15-30)$ residual topography component, consistent with $[9,41]$.

\section{Geodynamical Model Comparisons}

To quantify the relative contributions to our revised power spectrum from shallow and deep mantle flow, we also apply the ARD procedure to two end-member geodynamical simulations (see Methods for further details, including model 
limitations): (i) a simulation that neglects density and thermal heterogeneity in the uppermost $300 \mathrm{~km}$ of the mantle, with viscosity dependent upon depth only, allowing us to quantify the first-order topographic expression of deeper mantle flow (Fig. 1c); and (ii) a simulation constrained by an estimate of lithospheric thickness (Supplementary Fig. 6), which allows for the inclusion of shallow density heterogeneity and thermal structure, with viscosity dependent on both depth and temperature, thereby accounting for shallow mantle flow and its interaction with the lithosphere (Fig. 1d). The predicted topography from the first simulation shares many characteristics with published studies [30, 31, 42, 8, 33], displaying long-wavelength topographic highs within the Pacific domain, Southern and Eastern Africa and the North Atlantic, with lows extending across Central and South America, Europe, North West Africa and Asia. Spectral power displays a clear peak at $l=2$, with a rapid drop off at higher $l$ (Fig. 1f). Predictions from the second simulation, where the effect of ocean-floor cooling is removed using the plate model of [41], closely resemble those of [39], with shorter-wavelength topographic features clearly visible. The Pacific domain, however, is generally associated with a topographic high, albeit with a broad low off the west coast of South America and more localised lows in the northeast Pacific. Large topographic highs are also visible in the western US, western Antarctica, East Africa, the South China Sea, eastern Asia and adjacent to Iceland, with major lows focused along the AAD, the South Atlantic, the southern Indian Ocean and southeast of Arabia. Spectral power also peaks at $l=2$ (Fig. 1f), but it does not drop off significantly at higher $l$ and, in general character, is more consistent with the oceanic residual topography spectrum, albeit displaying larger amplitudes as identified by [39]. Both simulations predict more power than the observational constraints at $l \leq 2$, with this discrepancy largest in the model incorporating shallow structure.

These predictive models are next sampled at the locations of point-wise and ship-track oceanic residual topography estimates, thus enabling fully-consistent comparisons with the observational constraints, whilst the ARD procedure is performed independently for each inversion. Strikingly, the simulated dataset without shallow structure shows a sharp drop-off beyond $l=8$ (Fig. 2): the topographic signal lies below the assumed noise level in the data and, accordingly, the ARD procedure determines that higher spherical harmonic degrees can be set to zero without affecting data fit. This is in partial support of arguments made by [32,44], although it is noteworthy that this does not occur when applied to the observational constraints, demonstrating the robustness of the inferred short-wavelength residual topography signal.

The overall characteristics of the residual topography constraints are generally consistent with the simulation incorporating shallow structure. In comparison to the simulation, the observational dataset displays significantly less power at $l=1$ (an offset of $\sim 0.6 \mathrm{~km}^{2}$ ), and slightly less power throughout the remainder of the spectrum, but the overall trend is well-matched, with the range of plausible models often overlapping (beyond $l=1$, the offset is consistently below $0.12 \mathrm{~km}^{2}$ ). When combined, these comparisons demonstrate that: (i) the $l=2$ component of residual topography is compatible with the $l=2$ component of our predictive models, implying a key role for deep-mantle flow in dictating Earth's topographic signature, consistent with [32, 44]; and (ii) although spectral power does decrease by an order of magnitude from $l=2$ to $l=30$, the short-wavelength components are a direct manifestation of lithospheric structure and uppermost mantle dynamics, supporting the conclusions of [9]. We therefore return to the standpoint that the 
long-wavelength components of residual topography, which are principally controlled by deep-mantle flow, dominate the spherical harmonic power spectrum. The shorter-wavelength components, dictated by lithospheric structure and uppermost mantle flow, are robust, albeit less significant, in terms of spectral power [38, 5]. Critically, the observational constraints support a crucial role for both deep and shallow mantle flow in dictating Earth's surface response.

\section{Isolating the Flow-Related Component of Residual Topography}

The observational constraints on residual topography utilised here are not solely a consequence of underlying mantle flow (i.e. they are not fully dynamic in origin). As stated previously, to account for the effects of ocean-floor cooling with age, an age-dependent theoretical plate model has been subtracted from the isostatically (crustal and sediment) corrected topography [41]. The same procedure was applied to our simulation that incorporates shallow structure, to generate a consistent synthetic estimate of residual topography and, thus, enable direct comparison with the observational constraints. However, by doing so, we are assuming that, in the oceans, lithospheric thickness (and density) varies as a function of ocean-floor-age only and, hence, are ignoring local deviations about this average behaviour that are apparent in Supplementary Fig. 6. Accordingly, the effect of anomalous, non-age-dependent lithospheric thickness variations are incorporated into the residual topography estimates. Such variability is likely isostatic in nature [46, 35] and not a direct manifestation of present-day mantle flow. Indeed, as stated by [41], observational constraints on residual topography represent an upper-bound on the flow-related dynamic topography component.

Given that our synthetic residual topography field is generally consistent with Earth's residual topography expression (correlation $=0.4)$, it is of interest to isolate the dynamic (flow-related) component in our simulation, in an attempt to better understand this partitioning on Earth. We do so using a simple approach, which assumes that lithospheric thickness variations are thermal in origin (poorly constrained compositional variations are neglected) and have an isostatic contribution that can be subtracted out (see Methods). Our approximation of the resulting dynamic topography field is illustrated in Supplementary Fig. 7: this displays clear differences to the model that omits shallow structure (cf. Fig. 1c). These differences are confirmed by an ARD-based inversion (solely within the oceanic realm at the locations of spot and ship-track measurements): the general character of the resulting dynamic topography power spectrum is broadly consistent with the residual topography spectrum, but distinct from the spectrum of the model neglecting shallow structure (Fig. 3a). This indicates that the interplay between upper mantle flow and the base of Earth's heterogeneous lithosphere plays a crucial role in generating dynamic topography and in controlling the character of the power spectrum.

Closer examination of these spectra reveals another important trend (Fig. $3 \mathrm{~b})$ : within the oceanic realm, at $l=2$, greater than $80 \%$ of the synthetic residual topography signal is related to mantle flow, as opposed to mapped lithospheric thickness variations. Conversely, at higher $l$ (particularly at $l \geq 15$ ), greater than $50 \%$ of the residual topography signal can be attributed to isostatic effects arising from non age-dependent variations in lithospheric thickness. Our approach 
Figures/Fig_3.pdf

Figure 3: Dynamic (flow-related) component of synthetic residual topography within the oceanic realm: (a) power spectra of residual (blue) and flow-related (green) synthetic topography predictions from the model that incorporates shallow structure, using inversions regularised with ARD [43, 49]. Plotting conventions are as described in caption to Fig. 2, with spectra from a simulation with no shallow structure, identical to Fig. 2, displayed for ease of comparison. In isolating the dynamic component of residual topography an estimate of isostatic contributions resulting from variations in lithospheric thickness has been removed from the original synthetic residual topography field. The ratio between the mean of both power spectra, displayed in (b), demonstrates important differences: at low $l$, and particularly at $l=2$ (vertical line), most of the synthetic residual topography is dynamic in origin, but greater than $50 \%$ of the signal can be attributed to mapped variations in lithospheric thickness at shorter wavelengths $(l \geq 15)$. 
is simplified, but these results are intriguing, implying that although oceanic residual topography measurements are a reasonable reflection of flow-related dynamic topography at long-wavelength, that is not the case at shorter-wavelengths. A corollary to this is that efforts to extract the shorter-wavelength components of flow-related dynamic topography from the observational record will only be successful if isostatic effects, arising from variations in the thickness and density of Earth's lithosphere, can be carefully isolated and removed. Doing so will require a comprehensive, multi-scale understanding of the structure and composition of Earth's lithosphere. It is therefore timely that recent studies are demonstrating significant progress in this endeavour [50].

\section{Online Content}

Any additional Methods, Supplementary Figures and Source Data are available in the online version of the paper; references unique to these sections appear only in the online paper.

\section{References}

[1] Davies, G. F. Dynamic Earth: plates, plumes and mantle convection (Cambridge University Press, 1999).

[2] Pekeris, C. L. Thermal convection in the interior of the Earth. Geophys. J. 3, 343-367 (1935).

[3] Parsons, B. \& Daly, S. The relationship between surface topography, gravity anomalies, and temperature structure of convection. J. Geophys. Res. 88, 1129-1144 (1983).

[4] Richards, M. A. \& Hager, B. H. Geoid anomalies in a dynamic Earth. J. Geophys. Res. 89, 5987-6002 (1984).

[5] Hager, B. H. \& Richards, A. M. Long-wavelength variations in Earth's geoid: Physical models and dynamical implications. Phil. Trans. Roy. Soc. London, Ser. A. 328, 309-327 (1989).

[6] Cazenave, A., Souriau, A. \& Dominh, K. Global coupling of Earth's surface topography with hotspots, geoid and mantle heterogeneities. Nature 340, 54-57 (1989).

[7] Braun, J. The many surface expressions of mantle dynamics. Nature Geoscience 3, 826-833 (2010).

[8] Flament, N., Gurnis, M. \& Muller, R. D. A review of observations and models of dynamic topography. Lithosphere (2013).

[9] Hoggard, M. J., White, N. \& Al-Attar, D. Global dynamic topography observations reveal limited influence of large-scale mantle flow. Nat. Geo. 9, 456-463 (2016).

[10] Lithgow-Bertelloni, C. \& Silver, P. G. Dynamic topography, plate driving forces and the African superswell. Nature 395, 269-272 (1998).

[11] Colli, L., Ghelichkhan, S. \& Bunge, H.-P. On the ratio of dynamic topography and gravity anomalies in a dynamic Earth. Geophys. Res. Lett. 43, 2510-2516 (2016).

[12] Burgess, P. M. \& Gurnis, M. Mechanisms for the formation of cratonic stratigraphic sequences. Earth Planet. Sci. Lett. 136, 647-663 (1995).

[13] Petersen, K. D., Nielsen, S., Clausen, O., Stephenson, R. \& Gerya, T. Small-scale mantle convection produces stratigraphic sequences in sedimentary basins. Science 329, 827-830 (2010).

[14] Shephard, G. E., Muller, R. D., Liu, L. \& Gurnis, M. Miocene drainage reversal of the Amazon River driven by plate-mantle interaction. Nature Geoscience 3, 870-875 (2010).

[15] Roberts, G. G. \& White, N. J. Estimating uplift rate histories from river profiles using african examples. J. Geophys. Res. 2010, B02406 (2010)

[16] Czarnota, K., Hoggard, M. J., White, N. \& Winterbourne, J. Spatial and temporal patterns of Cenozoic dynamic topography around Australia. Geochem. Geophys. Geosys. 14, 634-658 (2013).

[17] Czarnota, K., Roberts, G. G., White, N. J. \& Fishwick, S. Spatial and temporal patterns of Australian dynamic topography from river profile modelling. J. Geophys. Res. 119, 1384-1419 (2014).

[18] Eakin, C., Lithgow-Bertelloni, C. \& Davila, F. M. Influence of Peruvian flat-subduction dynamics on the evolution of western Amazonia. Earth Planet. Sci. Lett. 404, 250-260 (2014).

[19] Mitrovica, J. X., Beaumont, C. \& Jarvis, G. T. Tilting of continental interiors by the dynamical effects of subduction. Tectonics 8, 1079-1094 (1989).

[20] Gurnis, M. Phanerozoic marine inundation of continents driven by dynamic topography above subducting slabs. Nature 364, 589-593 (1993).

[21] Moucha, R. et al. Dynamic topography and long-term sea-level variations: There is no such thing as a stable continental platform. Earth Planet. Sci. Lett. 271, 101-108 (2008).

[22] Crameri, F. \& Lithgow-Bertelloni, C. Abrupt upper-plate tilting during slab-transition-zone collision. Tectonophys. 746, 199-211 (2018).

[23] Cao, W., Flament, N., Zahirovic, S., Williams, S. \& Muller, R. D. The interplay of dynamic topography and eustasy on continental flooding in the late Paleozoic. Tectonophysics In Press. (2019). 
24] Molnar, P. \& England, P. Late Cenozoic uplift of mountain ranges and global climate change: chicken and egg? Nature 346, 29-34 (1990).

[25] Crough, S. T. Hotspot swells. Ann. Rev. Earth Planet. Sci. 11, 165-193 (1983).

[26] Panasyuk, S. V. \& Hager, B. H. Models of isostatic and dynamic topography, geoid anomalies, and their uncertainties. J. Geophys. Res. 105, 28199-28209 (2000).

[27] Kaban, M. K., Schwintzer, P., Artemieva, I. M. \& Mooney, W. D. Density of the continental roots: Compositional and thermal contributions. Earth Planet. Sci. Lett. 209, 53-69 (2003).

[28] Guerri, M., Cammarano, F. \& Tackley, P. J. Modelling Earth's surface topography: decomposition of the static and dynamic components. Phys. Earth Planet. Int. 261, 172-186 (2016).

[29] Ricard, Y., Richards, M. A., Lithgow-Bertelloni, C. \& LeStunff, Y. A geodynamic model of mantle mass heterogeneities. J. Geophys Res. 98, 21895-21909 (1993).

[30] Steinberger, B. Effects of latent heat release at phase boundaries on the flow in the Earth's mantle, phase boundary topography and the dynamic topography at the Earth's surface. Phys. Earth Planet. Int. 164 (2007).

[31] Conrad, C. \& Husson, L. Influence of dynamic topography on sea level and its rate of change. Lithosphere 1, 110-120 (2009).

[32] Yang, T. \& Gurnis, M. Dynamic topography, gravity and the role of lateral viscosity variations from inversion of global mantle flow. Geophys. J. Int. 207, 1186-1201 (2016).

[33] Rubey, M. et al. Global patterns in Earth's dynamic topography since the Jurassic: the role of subducted slabs. Solid Earth 8, 899-919 (2017).

[34] Colli, L., Ghelichkhan, S., Bunge, H.-P. \& Oeser, J. Retrodictions of Mid Paleogene mantle flow and dynamic topography in the Atlantic region from compressible high resolution adjoint mantle convection models: Sensitivity to deep mantle viscosity and tomographic input model. Gondwana Res. 53, 252-272 (2018).

[35] Eakin, C. \& Lithgow-Bertelloni, C. An overview of dynamic topography: The influence of mantle circulation on surface topography and landscape. Mountains, Climate and biodiversity $\mathbf{3 7}$ (2018).

[36] Molnar, P., England, P. C. \& Jones, C. H. Mantle dynamics, isostasy, and the support of high terrain. J. Geophys. Res. 120, 1932-1957 (2015).

[37] Crosby, A. G. \& McKenzie, D. An analysis of young ocean depth, gravity and global residual topography. Geophys. J. Int. 178, 1198-1219 (2009).

[38] Hager, B. H. Subducted slabs and the geoid: Constraints on mantle rheology and flow. J. Geophys. Res. 89, 6003-6015 (1984).

[39] Steinberger, B. Topography caused by mantle density variations: observation-based estimates and models derived from tomography and lithosphere thickness. Geophys. J. Int. 205, 604-621 (2016).

[40] Laske, G., Masters, G., Ma, Z. \& Pasyanos, M. Update on CRUST1.0 - A 1-degree Global Model of Earths Crust. Geophysical Research Abstracts Abstract EGU2013-2658 (2013).

[41] Hoggard, M. J., Winterbourne, J., Czarnota, K. \& White, N. Oceanic residual depth measurements, the plate cooling model, and global dynamic topography. J. Geophys. Res. 122, 2328-2372 (2017).

[42] Spasojevic, S. \& Gurnis, M. Sea level and vertical motion of continents from dynamic Earth models since the Late Cretaceous. Am. Assoc. Petrol. Geol. Bulletin 10, 2037-2064 (2012).

[43] Valentine, A. P. \& Sambridge, M. Optimal regularisation for a class of linear inverse problem. Geophys. J. Int. 215, 1003-1021 (2018).

[44] Yang, T., Moresi, L., Muller, R. D. \& Gurnis, M. Oceanic residual topography agrees with mantle flow predictions at long wavelengths. Geophys. Res. Lett. 44, 10896-10906 (2017).

[45] Watkins, C. E. \& Conrad, C. P. Constraints on dynamic topography from asymmetric subsidence of the mid-ocean ridges. Earth Planet. Sci. Lett. 484, 264-275 (2018).

[46] Arnould, M., Coltice, N., Flament, N., Seigneour, V. \& Muller, R. D. On the scales of dynamic topography in whole-mantle convection models. Geochem. Geophys. Geosys. 19, 1-24 (2018).

[47] Steinberger, B., Conrad, C. P., Osei Tutu, A. \& Hoggard, M. J. On the amplitude of dynamic topography at spherical harmonic degree two. Tectonophys. In Press. (2019).

[48] Tarantola, A. \& Valette, B. Generalised non-linear inverse problems solved using the least squares criterion. Rev. Geophys. Space Phys. 20, 219-232 (1982).

[49] MacKay, D. Bayesian interpolation. Neural. Comput. 4, 415-447 (1992).

[50] Afonso, J. C., Salajegheh, F., Szwillus, W., Ebbing, J. \& Gaina, C. A global reference model of the lithosphere and upper mantle from joint inversion and analysis of multiple data sets. Geophys. J. Int. 1602-1628 (2019).

\section{Acknowledgments}

We acknowledge support from the Australian Research Council, under grant numbers FT140101262, DP170100058 (both DRD) and DE180100040 (APV). MJH acknowledges support from the National Aeronautics and Space Administration grant NNX17AE17G. Numerical simulations were undertaken on the NCI National Facility in Canberra, Australia, which is supported by the Australian Commonwealth Government. We thank two anonymous reviewers for careful and constructive comments during the review process. 


\section{${ }_{312}$ Author Contributions}

DRD conceived this study, designed, setup and processed the geodynamical simulations and integrated all interdisciplinary components. APV developed the tools utilised for spherical harmonic analyses and supported with their application and interpretation. SCK, CRW and DRD lead the development and validation of Fluidity. NR produced the lithospheric thickness model, MJH provided the observational constraints on residual topography, whilst CME provided

317 insight on dynamic topography implications and comparisons across various datasets and models. DRD and APV wrote 318 the paper, following discussion with, and contributions from, all authors.

\section{${ }_{319}$ Author Information}

320 The authors declare no competing financial interests.

\section{Additional Information}

322 Supplementary information is available for this paper at:

323 Reprints and permissions information is available at www.nature.com/reprints.

324 Correspondence and requests for materials should be addressed to DRD (Rhodri.Davies@anu.edu.au). 


\section{Methods}

Global Mantle Flow Simulations - Approach \& Limitations: We focus on two end-member simulations of global mantle flow. In the first, lateral variations in density and viscosity are ignored in the uppermost mantle (above $300 \mathrm{~km}$ depth), thus allowing us to quantify the first-order topographic expression of deeper mantle flow. In the second, we account for the effects of shallow mantle flow and its interaction with the lithosphere, by incorporating variations in density and temperature (and the associated variations in viscosity) for the entire convecting mantle and lithosphere.

We solve the equations governing instantaneous mantle convection (i.e. the present-day flow-field is computed in the context of prescribed density and rheological variations) inside a spherical shell, using a modified version of Fluidity $[51,52,53,54]$, recently validated against a range of analytical solutions and benchmarked against published results from alternative spherical shell mantle convection codes $[55,56,57]$. In our simulations, the inner radius corresponds to the core-mantle-boundary (CMB) and the outer radius to Earth's surface. Free-slip mechanical boundary conditions are specified at each boundary. Consistent with a number of previous models $[8,39$, 47], we assume incompressibility and the Boussinesq approximation, with phase transitions neglected. Models employ a fixed icosahedral mesh with a lateral resolution of $\sim 50 \mathrm{~km}$ at the surface. This is extruded in the radial direction, with radial spacing increasing linearly from $10 \mathrm{~km}$ at the surface to $100 \mathrm{~km}$ at the CMB.

In our first model, density anomalies below $300 \mathrm{~km}$ depth are derived from the shear-wave tomography model S40RTS [58], using the conversion factor $\mathrm{d} \ln \rho / \mathrm{d} \ln V_{S}=0.2$. Density variations above this depth are neglected. This model includes a simple depth-dependent viscosity, with 5 layers, as illustrated in Supplementary Fig. 8(a). Dynamic topography is computed from radial stresses, $\tau_{r r}$, at the surface via: $h=\tau_{r r} /\left(\Delta \rho_{\text {ext }} \cdot g\right)$, where $\Delta \rho_{\text {ext }}$ is the density contrast between uppermost mantle density and air (continents: $3300 \mathrm{~kg} \mathrm{~m}^{-3}$ ) or water (oceans: $2300 \mathrm{~kg} \mathrm{~m}^{-3}$ ), and $g$ $\left(9.81 \mathrm{~m} \mathrm{~s}^{-2}\right)$ is the gravitational acceleration (continents are defined as regions where the age-grid of [59] is undefined). The surface topography computed from this model is therefore a direct manifestation of flow related normal stresses (i.e. it is solely dynamic in origin). As illustrated in Fig. 1(c), topographic predictions share many characteristics with several published predictive models $[30,31,42,8,33]$.

In our second model, we account for the first-order effects of lithospheric thickness, which allows us to approximate density anomalies above $300 \mathrm{~km}$ depth, leading to more complete computations of mantle flow and the associated surface topography. Lithospheric thickness is estimated using the method first described in [60], in which the depth-averaged velocity in the upper mantle (to a depth of $350 \mathrm{~km}$ ) is assumed to be proportional to lithospheric thickness. In this case, the upper mantle Sv model SL2013sv [61] is used as input, with the resulting estimate of lithospheric thickness illustrated in Supplementary Fig. 6. Our method reproduces the first-order characteristics of other global lithospheric thickness models $[62,50]$, predicting that the lithosphere is generally thin beneath young oceans and thicker beneath older oceans and continents. Within the oceans, there is a general trend of increasing lithospheric thickness with age. Within the continents, cratonic regions generally have thicknesses of 250-300 km, with thinner regions found near areas of recent or ongoing subduction or rifting (e.g. western USA and eastern Asia, northeastern Africa). We note that 
lithospheric thickness models derived from SL2013sv have already been successfully applied in global models [39] and regional studies of the North American lithosphere [63].

The conversion of seismic velocity to density inside the lithosphere is complicated by contrasting thermal and chemical effects. We take a simple approach, first prescribing a thermal structure within the lithosphere, based upon an error function temperature profile that corresponds to the lithospheric thickness model (assuming a thermal diffusivity, $\kappa=$ $\left.7.5 \times 10^{-7} \mathrm{~m}^{2} \mathrm{~s}^{-1}\right)$. Temperatures are subsequently converted to density using a linearised equation of state: $\rho=$ $\rho_{0}(1-\alpha \Delta T)$, where $\rho_{0}=3300 \mathrm{~kg} \mathrm{~m} \mathrm{~m}^{-3}$ and $\alpha=2.5 \times 10^{-5} \mathrm{~K}^{-1}$. Continental roots are seismically fast, but are likely neutrally buoyant $[64,65]$. Accordingly, we follow the approach of [66] and set density anomalies of the mantle's upper $300 \mathrm{~km}$ smoothly to zero beneath continental regions, defined as those regions where $\mathrm{d} \ln V_{S}>4 \%$ at $100 \mathrm{~km}$ depth in the SL2013sv model [61]. Masking out continental roots introduces a compositional anomaly, $c$, which is unity everywhere below $300 \mathrm{~km}$ and tends toward zero inside old continental regions. Sub-lithospheric density anomalies are derived from S40RTS [58], consistent with our first model. Sub-lithospheric temperatures are also derived directly from tomography [58], using the conversion factor $\Delta T / \mathrm{d} \ln V_{S}=-80 \mathrm{~K} / \%$. For this model, we assume a temperature- and depth-dependent viscosity, following the relation: $\mu_{r} \cdot \exp \left[E\left(0.5-T^{*}\right)\right]$, where $T^{*}$ is the non-dimensional temperature, $E=18.42$, whilst $\mu_{r}$ varies with depth and is set to ensure a mean viscosity consistent with the first model. Resulting viscosities are displayed in Supplementary Fig. 8(b).

The inclusion of shallow structure, via a lithospheric thickness estimate, implies that the topography computed from radial stresses at the surface incorporates subsidence of the ocean-floor. Accordingly, to allow for direct quantitative comparisons with our residual topography dataset, the effect of ocean-floor subsidence is subtracted out using an identical plate model and ocean-floor age grid. Where the age-grid is undefined (i.e. on continents) we follow [39] and assume an age of $175 \mathrm{Myr}$, such that synthetic topography predictions display no dramatic steps across the continent-ocean boundary. Resulting topographies are subsequently adjusted to ensure a global mean of zero. We note that the resultant synthetic topography prediction incorporates the effects of non-age-dependent lithospheric thickness variations, as is the case for the observational constraints, the significance of which is examined in the main text. As illustrated in Fig. 1(d), topographic predictions from the second model closely resemble those of [39], who also incorporate shallow mantle and lithospheric structure.

Whilst this paper focuses on the aforementioned cases, we have analysed a series of simulations, with systematically increasing complexity. The starting point is the first model above, which neglects all (density and rheological) heterogeneity above $300 \mathrm{~km}$ depth. To this, we have added lateral (temperature- and pressure-dependent) variations in viscosity throughout the computational domain. The resulting topographic field is illustrated in Supplementary Fig. 9(c), with the difference to the starting model highlighted in Supplementary Fig. 9(d). Spectral decomposition of both models (Supplementary Fig. 9b, red lines) yields similar results, suggesting that topographic predictions are only weakly sensitive to lateral variations in viscosity within the convecting mantle and lithosphere. We note that this result is at odds with [67], the reasons for which require further investigation. In Supplementary Fig. 9(f), we illustrate 
the difference between this model and a model where we incorporate shallow density heterogeneity based upon our global estimate of lithospheric thickness (Supplementary Fig. 9e). The difference field closely resembles the full model (Supplementary Fig. 9e), demonstrating the dominant role of shallow structure in generating topography, as expected from the sensitivity kernels that illustrate how effective density anomalies at different depths and spherical harmonic degrees are at creating topography $[38,5,11,39]$. In Supplementary Fig. $9(\mathrm{~g})$, we explore the effect of a different surface velocity boundary condition, prescribing present-day plate velocities from the dataset of [68]. As noted by [39], this change has an important effect at long wavelengths (increasing spectral power marginally at $l<5$ ), causing a decrease in topography beneath mid-ocean ridges, particularly at fast spreading centres (e.g. the East Pacific rise) and an increase in topography within the Western and Central Pacific. It remains unclear whether prescribed or free-slip boundary conditions are the most suitable for simulations of this nature: if plate motions are prescribed but are inconsistent with the forces acting on these plates, the computed topography may be inappropriate, hence our decision to focus on the simulation with free-slip boundary conditions herein.

We emphasise that fine details of the predicted topography in our simulations and their associated power spectra are sensitive to several model parameters. These include: (i) the depth- and lateral-dependence of mantle viscosity, which remain poorly constrained $[69,70,71,72]$, and may influence both coupling between upper and lower mantle and the transmission of stress across the asthenosphere to the lithosphere; (ii) the seismic tomography model used as basis for defining the mantle's density and thermal structure - although tomographic models now show broad similarity in the distribution of heterogeneity at a large-scale [73], they differ in amplitude and in the distribution of smaller-scale heterogeneity; (iii) our approach for converting seismic velocity to density and temperature, noting that the constant conversion factor used does not account for the non-linear sensitivity of seismic velocity to pressure, temperature, composition and phase [74, 75, 76, 77, 78, 79]; and (iv) the lithospheric thickness estimate utilised, variations to which will modify how mantle flow interacts with shallow structure [39, 62]. Nonetheless, our models are based upon a reasonable set of parameters that allow us to illustrate the likely roles of shallow and deep mantle flow in generating Earth's surface response: our raw numerical predictions are generally consistent with time-dependent and instantaneous flow models from published studies $[8,39]$ as they have been executed in a similar parameter space. With our current understanding of the uncertainties surrounding these parameters and the associated sensitivities [30,39, 47], it appears unlikely that our conclusions would be modified substantially. However, we acknowledge that this should be explored, in detail, in the future, in both time-dependent models of mantle flow that exploit, for example, time-integrated histories of Phanerozoic plate subduction [8] and present-day tomographic constraints [42, 34], and instantaneous flow models that better-account for tomographic and mineral physics uncertainties.

Extraction of Dynamic (Flow-Related) Component from Synthetic Residual Topography Field: The definition of dynamic topography excludes topography isostatically supported through crustal thickness variations. However, it is less clear whether or not topography supported by density anomalies within the mantle lithosphere, or lithospheric thickness variations, should be included $[9,41,39,35]$. Both the observational constraints on residual topography and the 
synthetic residual topography estimates utilised herein (for the model with shallow structure) incorporate topographic contributions arising from lithospheric thickness and density variations (i.e. deviations from a plate cooling model) as well as present-day mantle flow (i.e. dynamic topography directly due to normal stresses imposed by underlying mantle flow). It is of interest to isolate the dynamic (flow-related) component in our synthetic residual topography field, in an attempt to better understand this partitioning on Earth. To do so, we take the following steps: (i) assuming that lithospheric thickness variations are thermal in origin, we invert our lithospheric thickness model for a map of lithospheric age, based upon the half-space cooling approximation (noting that continental regions are assigned a constant age of 175 Myr); (ii) under the assumption that lithospheric thickness variations make an isostatic contribution towards residual topography, we subtract point-wise estimates of subsidence based upon this lithospheric age map and the following relationship between age and depth: $d=2.6+0.25$ (age $)^{1 / 2}$ (with age in Myr), with the resulting global topography field adjusted to ensure a mean of zero. This differs to subtracting an ocean-floor-age-dependent plate model from our topographic prediction, and yields an approximation to the flow-related component of topography, illustrated in Supplementary Fig. 7. The associated spherical harmonic spectral decomposition (carried out solely within the oceanic realm at the locations of spot and ship-track observational constraints) is displayed in Fig. 3 of the main manuscript.

Computation of Power Spectra and Uncertainties: Our approach is based on that set out in [9]. We assume that the topographic signal can be represented in terms of a spherical harmonic expansion up to degree $l_{\max }$, omitting the spherically-symmetric (degree-0) term. We therefore write $f(\theta, \phi)=\sum_{l=1}^{l \max } \sum_{m=-l}^{l} c_{l m} \mathcal{Y}_{l m}(\theta, \phi)$, where $\mathcal{Y}_{l m}$ is a real surface spherical harmonic as defined in Section B.6 of [80]; note that these are normalised to have unit power. We then perform a regularised least-squares inversion to recover the $\left[\left(l_{\max }+1\right)^{2}-1\right]$ coefficients of this expansion. In all cases, we invert for a model up to maximum spherical harmonic degree $l_{\max }=50$, but plot results only up to $l=30$. In doing so, we aim to minimise effects arising from spectral leakage [81].

We follow [48], and note that least-squares inversion can be framed as a Bayesian inference procedure. We use $\mathbf{m}$ to denote a vector containing all the coefficients $c_{l m}$. Our prior probability distribution for $\mathbf{m}$ - that is, our state of knowledge before seeing any data - has Gaussian form, with zero mean, and is characterised by a prior model covariance matrix $\mathbf{C}_{\mathbf{m}}$. Our vector of observations, $\mathbf{d}$, is assumed to be subject to random noise described by a zero-mean Gaussian with covariance matrix $\mathbf{C}_{\mathbf{d}}$. For this study, we assume that the noise has no spatial correlations, so that the covariance matrix has non-zero elements only on the leading diagonal; we base these on the uncertainty estimates reported by [9] (including in cases where the 'data' being inverted are the output of a numerical simulation). Given these assumptions, the information obtained from the observations leads us to a posterior probability distribution of Gaussian form centred on $\mathbf{m}=\left(\mathbf{A}^{\mathbf{T}} \mathbf{C}_{\mathbf{d}}^{-\mathbf{1}} \mathbf{A}+\mathbf{C}_{\mathbf{m}}^{-\mathbf{1}}\right)^{-\mathbf{1}} \mathbf{A}^{\mathbf{T}} \mathbf{C}_{\mathbf{d}}^{-\mathbf{1}} \mathbf{d}$, with covariance matrix $\left(\mathbf{A}^{\mathbf{T}} \mathbf{C}_{\mathbf{d}}^{-\mathbf{1}} \mathbf{A}+\mathbf{C}_{\mathbf{m}}^{-\mathbf{1}}\right)^{-\mathbf{1}}$. The matrix $\mathbf{A}$ here is defined such that $[\mathbf{A}]_{i j}=\mathcal{Y}_{l_{j} m_{j}}\left(\theta_{i}, \phi_{i}\right)$, where $\left(\theta_{i}, \phi_{i}\right)$ represents the location associated with the $i$-th element of $\mathbf{d}$, and where $l_{j}$ and $m_{j}$ denote the degree and order of the spherical harmonic coefficient represented by the $j$-th element of $\mathbf{m}$. The power at degree $l$ is then defined as $P_{l}=\sum_{m=-l}^{l} c_{l m}^{2}$; this is the quantity we plot when we show spectra.

To complete the specification of our inversion procedure, it is necessary to define $\mathbf{C}_{\mathbf{m}}$. Our approach here builds on 
[43], and a full discussion of the issues surrounding regularisation can be found in that study. We employ two different forms of model covariance matrix in this paper. The first is as used in [9], and is most conveniently specified by stating a parametric form for the inverse covariance matrix, $\mathbf{C}_{\mathbf{m}}^{-\mathbf{1}}=\alpha^{2} \mathbf{I}+\beta^{2} \mathbf{H}$, where $\alpha$ (overall power term) and $\beta$ (gradient term) are tuneable hyperparameters, $\mathbf{I}$ is an identity matrix, and where $[\mathbf{H}]_{i j}=l_{i}\left(l_{i}+1\right) \delta_{i j}$. As described in $[9]$, this $\mathbf{H}$ penalises steep gradients in the recovered field. We refer to this form of covariance matrix as 'Tikhonov-style'. Results from inversions of this nature are described in the Supplement. The second form of regularisation employed, which we refer to as Automatic Relevance Determination (ARD) following [49], amounts to choosing $\left[\mathbf{C}_{\mathbf{m}}^{-1}\right]_{i j}=\xi_{l_{i}} \delta_{i j}$, with $l_{\max }$ tuneable hyperparameters $\xi_{i}$. Thus, all spherical harmonics of a given degree (i.e. all $m$ for fixed $l$ ) are treated identically, but no relationships are enforced between separate degrees. In both cases, a hierarchical Bayesian approach described fully in [43] allows us to assess the probability that any particular choice of hyperparameters is consistent with the data; we can then identify the most-probable hyperparameters to use in inversion. Results utilising this form of inversion, using point-wise and ship-track locations, are included in the main manuscript.

The posterior distribution provides insight into the extent to which the coefficients $c_{l m}$ are constrained by the data. While the posterior distribution on coefficients has Gaussian form, the power spectrum depends on the squared coefficients, and its posterior follows a generalized- $\chi^{2}$ (i.e., non-Gaussian) distribution. This has a number of counter-intuitive features, including the fact that the most-probable spectrum for a given dataset is not necessarily similar to the spectrum obtained from the most-probable set of coefficients. To provide a readily-understood quantification of uncertainty on spectra produced using Tikhonov-style regularisation, we: (i) generate 1,000 representative hyperparameter pairs $(\alpha, \beta)$ by sampling the hyperparameter probability distribution as described in [43]; (ii) perform an inversion of the dataset for each hyperparameter pair, to obtain a posterior Gaussian distribution for each; (iii) generate 100,000 random samples from each of these posterior distributions; (iv) convert each of these to a power spectrum; and (v) for each spherical harmonic degree, show the ranges spanned by the central $99 \%$ of the full set of samples, and the central $50 \%$ (i.e. the inter-quartile range). Steps (i) and (ii) ensure that our error bars take regularisation uncertainty into account. For ARD regularisation, we perform a similar process, except that we approximate the hyperparameter probability distribution using a multidimensional Gaussian with covariance inferred from the curvature of the true distribution at its peak (as discussed in [43]). This avoids practical difficulties associated with sampling an arbitrary multidimensional distribution.

\section{References}

[51] Davies, D. R., Wilson, C. R. \& Kramer, S. C. Fluidity: a fully unstructured anisotropic adaptive mesh computational modelling framework for geodynamics. Geochem. Geophys. Geosys. 120, Q06001 (2011).

[52] Kramer, S. C., Wilson, C. R. \& Davies, D. R. An implicit free-surface algorithm for geodynamical simulations. Phys. Earth Planet. Int. 194, 25-37 (2012).

[53] Davies, D. R. \& Rawlinson, N. On the origin of recent intra-plate volcanism in Australia. Geology 42, 1031-1034 (2014).

[54] Davies, D. R., Le Voci, G., Goes, S., Kramer, S. C. \& Wilson, C. R. The mantle wedge's transient 3-D flow regime and thermal structure. Geochem. Geophys. Geosys. 17, 78-100 (2016).

[55] Zhong, S., McNamara, A., Tan, E., Moresi, L. \& Gurnis, M. A benchmark study on mantle convection in a 3-D spherical shell using CitcomS. Geochem. Geophys. Geosys. 9, Q10017 (2008).

[56] Tackley, P. J. Modelling compressible mantle convection with large viscosity contrasts in a three-dimensional spherical shell using the Yin-Yang grid. Phys. Earth Planet. Int. 171, 7-18 (2008).

[57] Davies, D. R. et al. A hierarchical mesh refinement technique for global 3D spherical mantle convection modelling. Geosci. Mod. Dev. 6, 1095-1107 (2013). 


\section{${ }_{546}$ Data Availability} (2011). (2013). Planet. Sci. 44, 107-138 (2016). (2015). Int. 138, 197-222 (2003). (2004). (2005). (2011). $1257-1260$ (1996).

\section{Code Availability}

58] Ritsema, J., van Heijst, H. J., Deuss, A. \& Woodhouse, J. H. S40RTS: a Degree-40 shear velocity model for the mantle from new Rayleigh wave dispersion, teleseismic traveltimes, and normal-mode splitting function measurements. Geophys. J. Int. 184, 1223-1236

[59] Richards, F. D., Hoggard, M. J., Cowton, L. R. \& White, N. J. Reassessing the thermal structure of oceanic lithosphere with revised global inventories of basement depths and heat flow measurements. J. Geophys. Res. 123, 9136-9161 (2018).

[60] Davies, D. R., Rawlinson, N., Iaffaldano, G. \& Campbell, I. H. Lithospheric controls on magma composition along Earth's longest continental hotspot track. Nature 525, 511-514 (2015).

61] Schaeffer, A. J. \& Lebedev, S. Global shear speed structure of the upper mantle and transition zone. Geophys. J. Int. 194, 417-449

[62] Steinberger, B. \& Becker, T. W. A comparison of lithospheric thickness models. Tectonophysics 746, 325-338 (2018).

[63] Kaban, M. K., Tesauro, M., Mooney, W. D. \& Cloetingh, S. A. P. L. Density, temperature, and composition of the north american lithospherenew insights from a joint analysis of seismic, gravity, and mineral physics data: 1 . density structure of the crust and upper mantle. Geochem. Geophys. Geosyst. 15, 4781-4807 (2014).

[64] Jordan, T. H. Composition and development of the continental tectosphere. Nature 274, 544-548 (1978).

[65] Griffin, W. L., O'Reilly, S. Y., Afonso, J. C. \& Begg, G. C. The composition and evolution of lithospheric mantle: a re-evaluation and its tectonic implications. J. Petrology 50, 1185-1204 (2009).

[66] Becker, T. W. On the effect of temperature and strain-rate dependent viscosity on global mantle flow, net rotation and plate-driving forces. Geophys. J. Int. 167, 943-957 (2006).

[67] Osei Tutu, A., Steinberger, B., Sobolev, S. V., Rogozhina, I. \& Popov, A. A. Effects of upper mantle heterogeneities on the lithospheric stress field and dynamic topography. Solid Earth 9, 649-668 (2018).

[68] Muller, R. D. et al. Ocean Basin Evolution and Global-Scale Plate Reorganization Events Since Pangea Breakup. Ann. Rev. Earth

[69] Rudolph, M. L., Lekik, V. \& Lithgow-Bertelloni, C. Viscosity jump in Earth's mid-mantle. Science 350, 1349-1352 (2015).

[70] Marquardt, H. \& Miyagi, L. Slab stagnation in the shallow lower mantle linked to an increase in mantle viscosity. Nat. Geo. 8, 311-314

[71] Lau, H. C. P. et al. Tital tomography constrains Earth's deep-mantle buoyancy. Nature 551, 321-326 (2017).

[72] Ballmer, M. D., Houser, C., Hernlund, J. W., Wentzcovitch, R. \& Hirose, K. Persistence of strong silica-enriched domains in the Earth's lower mantle. Nat. Geo. 10, 236-241 (2017).

[73] Lekic, V., Cottaar, S., Dziewonski, A. \& Romanowicz, B. Cluster analysis of global lower mantle tomography: A new class of structure and implications for chemical heterogeneity. Earth Planet. Sci. Lett. 357, 68-77 (2011).

[74] Cammarano, F., Goes, S., Vacher, P. \& Giardini, D. Inferring upper-mantle temperatures from seismic velocities. Phys. Earth Planet.

[75] Goes, S., Cammarano, F. \& Hansen, U. Synthetic seismic signature of thermal mantle plumes. Earth Planet. Sci. Lett. 218, 403-419

[76] Stixrude, L. \& Lithgow-Bertelloni, C. Thermodynamics of mantle minerals - I. Physical properties. Geophys. J. Int. 162, 610-632

[77] Stixrude, L. \& Lithgow-Bertelloni, C. Thermodynamics of mantle minerals - II. Phase Equilibria. Geophys. J. Int. 184, 1180-1213

[78] Styles, E., Davies, D. R. \& Goes, S. Mapping spherical seismic into physical structure: biases from 3-D phase-transition and thermal boundary-layer heterogeneity. Geophys. J. Int. 184, 1371-1378 (2011).

[79] Davies, D. R. et al. Reconciling dynamic and seismic models of Earth's lower mantle: the dominant role of thermal heterogeneity. Earth Planet. Sci. Lett. 353-354, 253-269 (2012).

[80] Dahlen, F. A. \& Tromp, J. Theoretical Global Seismology (Princeton University Press, Princeton, 1998).

[81] Trampert, J. \& Snieder, R. Model estimations biased by truncated expansions: Possible artifacts in seismic tomography. Science 271,

The compilation of observational constraints on residual topography utilised herein, which builds on the database and methodology of [41], is available at https://github.com/drhodrid/Davies_etal_NGeo_2019_Datasets. Synthetic topography predictions from our geodynamical simulations are also included.

Fluidity is available under the GNU Lesser General Public License (LGPL). The source code and manual can be found at: http://fluidityproject.org. The optimal regularisation routines utilised for our spherical harmonic analyses are available from: https://github.com/valentineap/optimal-regularisation. 


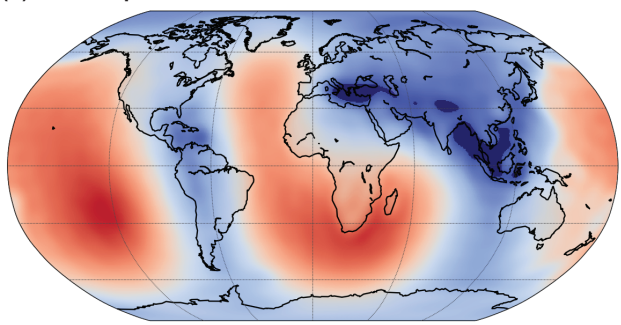

$-1.5 \mathrm{~km}$

(c) Instantaneous-flow simulation: no shallow structure

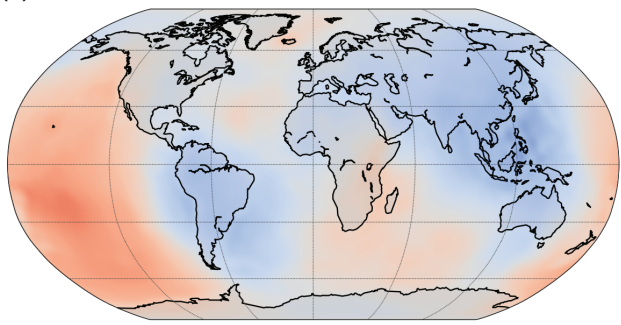

(e) Power spectra (previous studies)

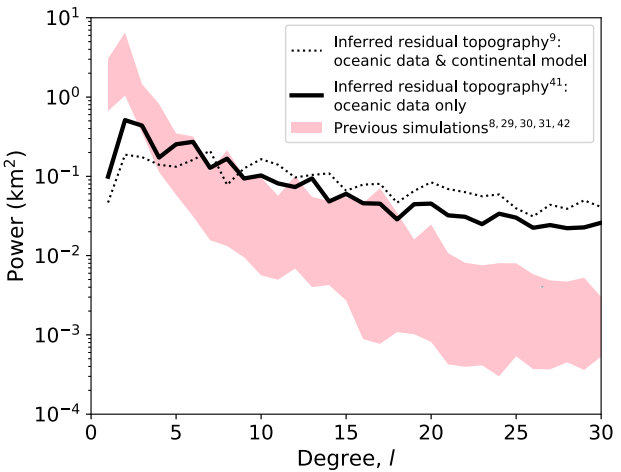

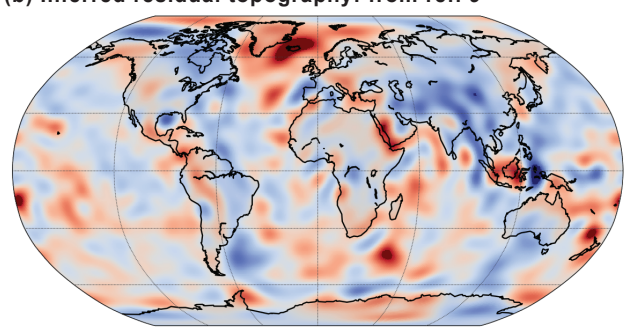

$+1.5 \mathrm{~km}$

(d) Instantaneous-flow simulation: with shallow structure

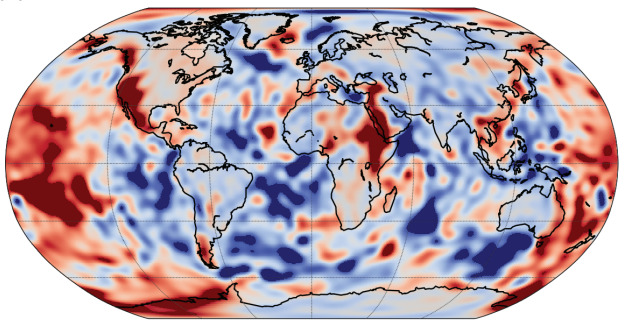

(f) Unregularized power spectra (this study)

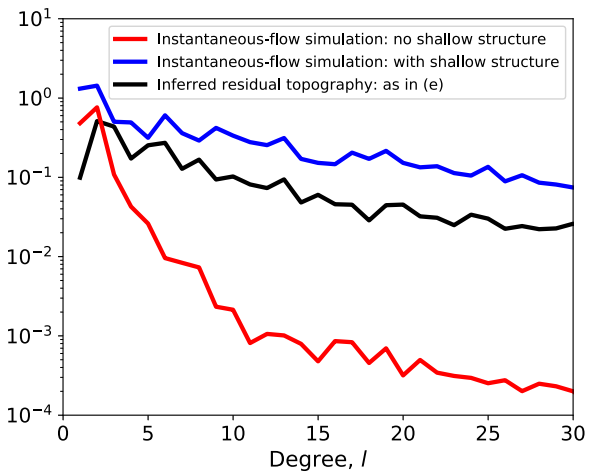




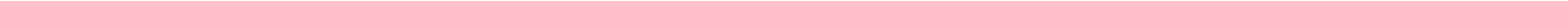

